\title{
Interactive Network Assessment Tool Using ArcGIS API for JavaScript
}

\author{
Robin Wendel \\ Interfaculty Department of Geoinformatics, University of Salzburg, Austria · robin.wendel@sbg.ac.at
}

Full paper double blind review

\begin{abstract}
Road safety is a core element of any bicycle promotion strategy. In order to gain an overview of the status quo, and to provide a basis for evidence-based decisions and measures, the quality of the road network in terms of safety needs to be assessed. For this, a webbased tool is introduced, which conceptually builds upon an indicator-based assessment model. Its applicability is demonstrated in a case study deploying the workflow on Austria's authoritative transportation network dataset GIP (Graphenintegrations-Plattform).
\end{abstract}

\section{Introduction}

Whenever bicycles are suggested as an alternative mode of transport, it is assumed that cycling is healthy, but dangerous compared to the travelled distance (JUHRA et al. 2012, BECK et al. 2007). Safety issues are the main reason for refusing bicycles as a serious option (LORENC et al. 2008). Researchers showed that these safety issues are related to the physical conditions of the road network (WEGMAN et al. 2012). Hence, the infrastructure must be designed in a manner that minimizes the risks for cyclists (TESCHKE et al. 2012). In order to gain an overview of the road safety conditions, and to provide a basis for evidencebased decisions and measures, an interactive and highly dynamic approach for the assessment of road networks is introduced.

This interactive network assessment is based on the indicator-based assessment model by LOIDL \& ZAGEL $(2010,2014)$. The basic idea of the model is to identify indicators (e.g. bicycle infrastructure, traffic volume), which describe the constitution of the road network and contribute to the explanatory value of a specific use case (e.g. bicycle safety). These indicators are then weighted, and result in an index value (weighted average). Until now, the assessment used previously defined input parameters and weights. The definition and calibration of those required much effort and expertise in spatial data modeling. Environmental differences (e.g. urban vs. rural) or individual preferences were difficult to consider.

The developed web-based tool facilitates an interactive manipulation and calibration of input parameters by non-GIS experts, and also allows for personalized settings. The respective results of any changes are visualized and controlled immediately online. With this, the original model can be adapted to a dynamic process, making the results more transparent and comprehensible. Additionally, it becomes obvious, which specific measures contribute to the quality of the respective road segment, and to what extent. This makes the tool well suited for planning and simulation purposes. 
The paper provides a generic description of the interactive network assessment workflow, and a draft of a corresponding web-based tool, fitting the special needs of a road network assessment in terms of bicycle safety. The tool provides the possibility of dynamically adjusting the weightings and simulating possible measures, while instantly giving visual feedback. By adapting the indicators and weightings, the approach can be modified to fit miscellaneous road network datasets and use cases.

The paper is structured in the following way: Initially the generic workflow of the interactive network assessment is described, which is then demonstrated in a case study on bicycle safety. After pointing out the used frameworks and data, the application development of the corresponding tool is explained in detail. Furthermore, the graphical user interface is illustrated. Finally, the results of the case study are discussed, the benefits of the tool are consolidated, and possible fields of application are proposed.

\section{Interactive Network Assessment Tool}

The interactive network assessment tool follows the logic of the original model (see Fig. 1). In a first step, information about a specific use case (e.g. bicycle safety) is collected. In order to identify significant indicators, literature is examined, and experts or users are interviewed $^{1}$. In a preprocessing step these indicators (e.g. bicycle infrastructure) and their value domains (e.g. bicycle way, bicycle lane) are modeled for every road segment in a network dataset $^{2}$. An indicator is defined on the basis of one or more available attributes, which are classified to value domains. These value domains are assigned a fixed weighting between 0 and 1, representing good or bad road characteristics for the corresponding use case. The processed dataset is then provided as web feature service (WFS) on a geo-data server.

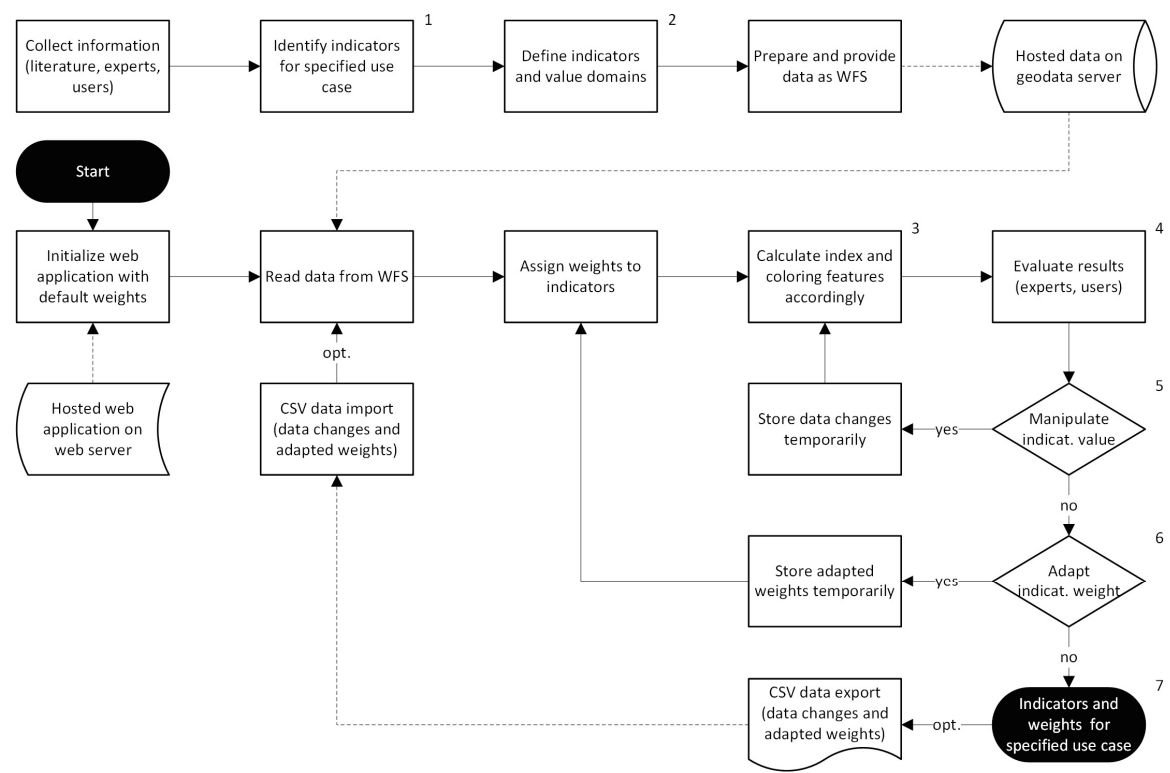

Fig. 1: Flowchart of the interactive network assessment tool with preprocessing steps 
The web tool subsequently reads the data from the WFS and assigns default weights to the indicators, enabling the index calculation and the appropriate coloring of the features ${ }^{3}$. The index coloring can be chosen freely, according to the range and meaning of the indicators (e.g. green to red). The index can now be adjusted by manipulating the indicator values ${ }^{5}$, and adapting the weightings for the indicators ${ }^{6}$ (see below). The results are displayed immediately, providing an overview of the implications ${ }^{4}$. Changing the weighting of one indicator to $100 \%$ allows the selected indicator to be reviewed. By examining all indicators in this way, unknown datasets can be explored and incorrect data can be traced and corrected immediately by manipulating the indicator value (e.g. by local knowledge). By weighting all indicators in compliance with the information found in literature, a first indicator set can be found. This can also be done subjectively by trying out different combinations or intersubjectively by consolidating the weightings gathered from experts or users who were given the tool and asked to provide their personal point of view (HARKEY et al. 1998). By fine-tuning the weightings the prominence of certain characteristics can be increased or decreased until a satisfying combination is found ${ }^{7}$.

Due to such a specific set of weightings, certain issues or concepts can be illustrated or explained. The dynamic approach helps finding these specified weightings by trying out various combinations. Constantly evaluating the outcomes enables counteractions whenever unwanted effects appear. At this stage experts (e.g. planners) get a qualitative overview of the road network data, reflecting a specific use case. The result can also be used as input for different geoprocessing purposes like routing, spatial simulations or location analysis.

\section{Case Study on Bicycle Safety}

The development of the interactive network assessment tool is exemplarily demonstrated in the case of bicycle safety. In a foregoing research, 13 indicators were found to be significant for the use case. The case study is built on Austria's authoritative, multimodal transportation network dataset GIP, which provided the geometry and attributive information for modeling the indicators. Therefore, attributes representing the physical conditions of the road network were classified to value domains, which were then weighted between 0 and 1 in relation to bicycle friendly and unfriendly conditions (see Tab. 1). Additionally, a point dataset was derived for visualizing the digitizing directions of the road segments as arrows. Both datasets were uploaded to a geodata server and provided as a WFS.

\begin{tabular}{|l|l|l|}
\hline Indicator & Value domain & Weighting \\
\hline Bicycle infrastructure & Bicycle way & Best $=0$ \\
& Bicycle and footway & Better $\approx 0.25$ \\
& Bicycle or bus lane & Good $\approx 0.5$ \\
& No bicycle infrastructure & Worst $=1$ \\
\hline Road category & Primary road & Worst $=1$ \\
& Secondary road & Worse $\approx 0.75$ \\
& Residential road & Good $\approx 0.5$ \\
& Calmed road & Better $\approx 0.25$ \\
& No road & Best $=0$ \\
\hline
\end{tabular}

Tab. 1:

Indicators with exemplary value domains and weightings 
The web-based tool was realized as an interactive and highly dynamic web map. The weightings were assigned to interdependent sliders. This allows the weightings to be adjusted, while always preserving $100 \%$ in total. The road geometry is colored accordingly to the respective index values, which are recalculated on every slider move. A possibility to manipulate indicator values was added (see Fig. 1), to enable the simulation of measures and the prediction of safety impacts.

\subsection{Frameworks and Data}

The application was developed using ESRI ArcGIS API for JavaScript, which brings functionality of geographical information systems to a simple web browser. This enables the usage of the application independently of an ArcGIS installation. However, it must be noted, that at least an ArcGIS Online subscription or an ArcGIS Server is necessary for hosting the network data when using this API.

The development of the application is based on the following components:

- ArcGIS API for JavaScript embeds a mapping functionality to a web application and retrieves information from a server through a REST API. Since it is based on JavaScript, the code runs in the browser instead of having it to run on the server, which provides a faster user experience. It is built on top of the Dojo Toolkit, making it independent of browser quirks (ESRI 2014a).

- Dojo Toolkit provides fast and lightweight JavaScript modules for building desktop and mobile web applications. The required capabilities can be loaded asynchronously, making the application load very fast. The widget library Dijit offers interactive web elements with the same look and feel in different browsers. DOM manipulations, event listing and effects are also part of the range of functions (THE DOJO FOUNDATION 2015).

- ArcGIS Online is a platform for creating and sharing interactive maps and apps. Therefore, predefined base-maps, tools, templates, and datasets are available, but also individual content can be uploaded and shared (ESRI 2014b).

The data used in the application is based on the GIP of Austria. The authoritative data is provided in the INTREST data format (IDF), which cannot be imported in most geospatial analysis tools. The IDF is a data exchange format for database dumps that need to be reconstructed first. The dataset includes comprehensive information on road traffic and physical parameters of the road network. For the case study, the road geometry and respective attributes describing the bicycle infrastructure, traffic volume, road category, speed limits, pavements, width, and number of lanes were extracted. Additionally, information for designated routes was joined from another authoritative dataset, gradient values were derived from a digital terrain model, and information on adjacent edges or crossing rails was added by spatial queries. Based on the compiled information, the indicators were defined.

\subsection{Application Development}

The application can be structured into four parts. First, the weightings are adapted with the sliders. Second, the weightings are derived as input parameters for the index calculation. Third, the road segments are rendered accordingly to the index values, and fourth, an overview of the data is provided and the simulation of measures is enabled. 


\subsubsection{Indicators Weighting}

The sliders are initialized as Dijit widgets. Each slider can have a value between 0 and $100 \%$ that stands for a weight of one indicator. This weight is calculated by dividing the slider value by 100 , resulting in a value between 0 and 1 . In total there are 13 sliders providing a weight for every indicator. The sum of the weights must always be 1 . This implies that if one slider is moved, the others need to be adjusted accordingly. Every time a slider is moved the index calculation and rendering is triggered to supply and display the current index values.

\subsubsection{Index Calculation}

An index value is calculated for every feature in the visible map extent, based on the indicator values und weights. The index is calculated using the following formula:

$$
\text { index }=\frac{\sum_{i=1}^{n} \text { indicator }_{i} * \text { weight }_{i}}{\sum_{i=1}^{n} \text { weight }_{i}}
$$

To apply this formula to datasets with varying completeness, it needs to be enhanced in order to handle missing data values. To achieve this, the index is calculated with only the available indicators for the respective road segment. Therefore, the corresponding weights are summarized. In order to normalize the weights again, they are divided by their sum, resulting in weights that are 1 again in sum.

\subsubsection{Feature Rendering}

The application includes two feature layers, which are symbolized differently based on the zoom level and chosen options. One layer is the actual road network symbolized as a line feature layer, and the other one as a point feature layer, indicating the digitization direction of the respective road segment with an arrow symbol. Based on the zoom level, the width of the lines and the size of the arrows are adapted, in order to preserve a good relation between visibility and usability. Due to event listeners the symbol of a hovered feature is replaced by a turquoise solid line symbol, making the actual extent of the feature visible. Simultaneously, a tooltip is shown, containing the corresponding index value.

The road segments are visualized as solid lines and colored from red to green according to the index values. Depending on the checked option buttons (see below) the segments are visualized differently. One-ways are symbolized with dash-dot-dot lines. Restrictions are colored grey, whereas pushing restrictions are shown as solid lines and access restrictions as dotted lines. Normally the index is calculated for both directions and weighted half each. One-ways are displayed if one of the directions is a one-way. Restrictions are displayed if both directions are restricted. In order to assess potential differences between both directions, it is possible to display only one direction (see Fig. 2). If "Index FT" (from-to) or "Index TF" (to-from) is selected, only the index for the respective direction (along or against the digitized direction) is calculated and visualized. In this case, arrows are displayed, showing the currently viewed direction. 

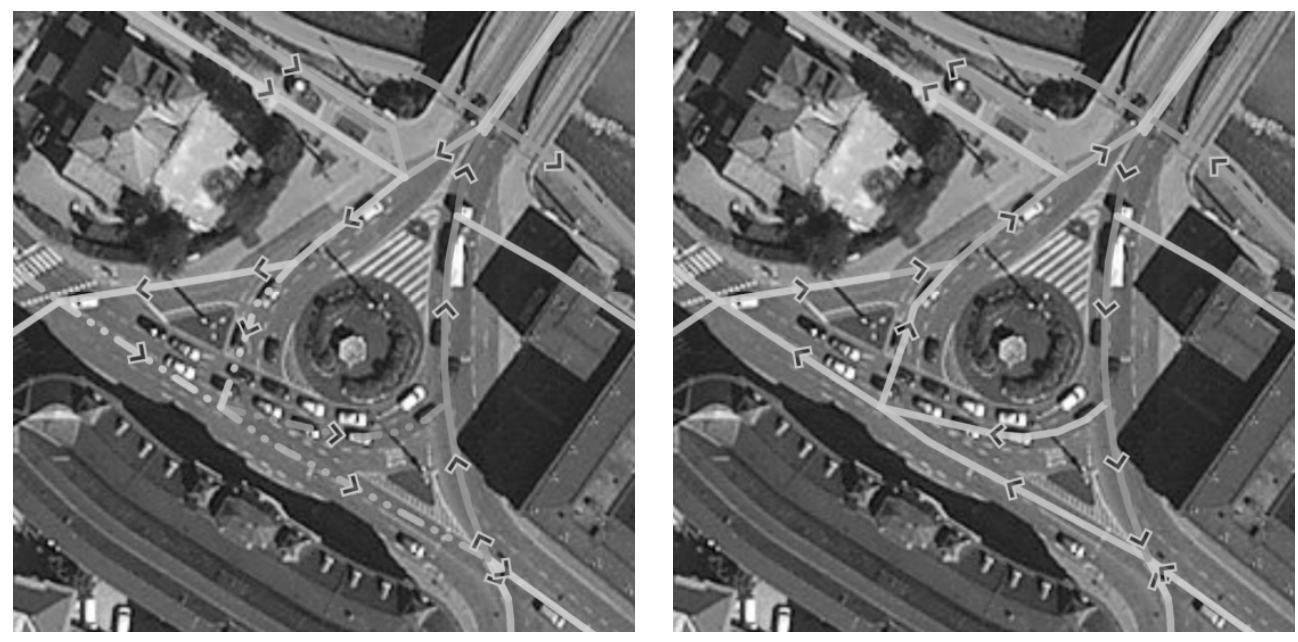

Fig. 2: Intersection rendered for different directions (road segments are colored according to the index values; one-ways have a different line symbol along the digitized direction; one-ways are restricted against the digitized direction)

The road network is symbolized using the ClassBreaksRenderer and the CartographicLineSymbol of the ArcGIS API. The class breaks are defined within the renderer, and each class break is assigned a unique symbol. Depending on which option buttons are checked, the index values are reclassified. For one-ways, the calculated index value is increased by two, for pushing restrictions four is returned, and for access restrictions five is returned. Depending on these values, every segment falls into a previously defined class. For roads without one-ways or restrictions, ten classes from 0.0 to 1.0 were defined and colored gradually from green to red with a SOLID line style. For one-ways, the same classes were defined from 2.0 to 3.0, but with a DASHDOTDOT line style. For road segments with missing index values, the classes -3.0 and -1.0 were defined. To distinguish at least between roads with and without one-ways, these classes were colored black, but with the different line styles. For restrictions the classes 4.0 and 5.0 are symbolized with the color grey and the line styles SOLID respectively DOT.

The arrows are symbolized using the PointRenderer of the ArcGIS API with an SVG path as symbol. The symbol is rotated, based on the angle at the middle point of a road segment. The point data was derived in a preprocessing step. Therefore a small piece of every segment was clipped through a $0.1 \mathrm{~m}$ buffer around the middle point. Then the angle was calculated using the following Python formula:

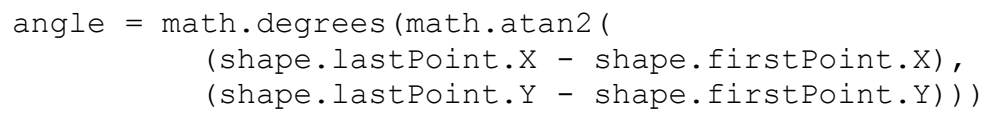

The results were joined back to the points. To prevent visual overlaps, the arrows are only displayed at higher zoom levels. Depending on the chosen direction, the symbol is rotated by 180 degrees. 


\subsubsection{Data Manipulation}

To provide an overview of the indicators and values, which contribute to the index value of a specific road segment, a popup window was implemented. Clicking on a road section opens an AttributeInspector window (see Fig. 3), showing all available indicators, and additionally allows the values to be edited. The coloring of the indicator values is based on the color scheme of the line features. Due to the fact that the indicator values have the same value range as the index values ( 0 to 1$)$, the perception of the implications is eased. In order

\begin{tabular}{|c|c|c|}
\hline \multicolumn{3}{|l|}{ Staatsbrücke } \\
\hline Bicycle Infrastructure FT: & Bicycle Way & 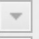 \\
\hline Bicycle Infrastructure TF: & Bicycle/Bus Lane & $\checkmark$ \\
\hline Traffic Volume FT: & $<7500$ & + \\
\hline Traffic Volume TF: & $<15000$ & - \\
\hline Designated Route FT: & National Route & $\checkmark$ \\
\hline Designated Route TF: & No & + \\
\hline Road Category: & Secondary Road & 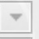 \\
\hline Max Speed FT: & $<70$ & $\checkmark$ \\
\hline Max Speed TF: & $<50$ & + \\
\hline Adjacent Edges FT: & 4 & - \\
\hline Adjacent Edges TF & $<=2$ & + \\
\hline Parking FT: & No & + \\
\hline Parking TF: & Yes & + \\
\hline Pavement: & Asphalt & 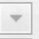 \\
\hline Width Lane: & $>2$ & 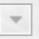 \\
\hline Gradient FT: & Downhill 2 & + \\
\hline Gradient TF: & Upslope 2 & - \\
\hline Rails: & No & + \\
\hline Number Lanes FT: & 2 & + \\
\hline Number Lanes TF: & 1 & 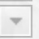 \\
\hline Land Use: & $\{$ Missing Value\} & $\nabla$ \\
\hline
\end{tabular}

Fig. 3: AttributeInspector window (indicator values are colored according to their impact) to change indicator values, a drop down list with available value domains is provided. When changing a value, the color of the chosen value is adapted. With that, the user gets a direct visual feedback about which indicator value contributes positively or negatively to the use case of bicycle safety, especially when simulating measures. Whenever the value of an indicator is changed, the index value of the respective road segment is recalculated, reclassified and colored accordingly. This enables the user to directly assess the impacts.

Values, which have been changed in the AttributeInspector window, are stored temporarily, and will be lost when the tool is reloaded. This feature enables the simulation of measures, without overwriting the initial dataset. During a session, the changes can be reset, exported, or stored permanently. By clicking the associated button, all changes are reset. By clicking "Edit Changes", a comma-separated list of the changes is produced. This list can be copied and imported in a future session, to restore the changes of previous sessions. By clicking "Apply Changes", the values in the web feature service are overwritten. This feature provides the possibility of correcting attributive errors in the dataset permanently, without changing the original data source. After reloading the tool these values will be the new standard values and cannot be automatically undone.

To allow subsequent geospatial analysis, a possibility to update the calculated index values to the web feature layer was implemented first. Due to timing issues, and a slow upload progress, this functionality was replaced by an ArcGIS model, which accepts the assessed weights as input parameters and recalculates the index fields quickly.

\subsection{Graphical User Interface}

An intuitive GUI design was targeted (Fig. 4), which was reduced to the most basic functions to reduce complexity. It allows experts, who are unfamiliar with scripting and geographic information systems, to assess road network attributes. A tooltip window was as- 
signed to each indicator, providing background information. The controls are separated into the sections "Options" and "Indicators". Additionally a home button was added to reach the initial extent and zoom level.

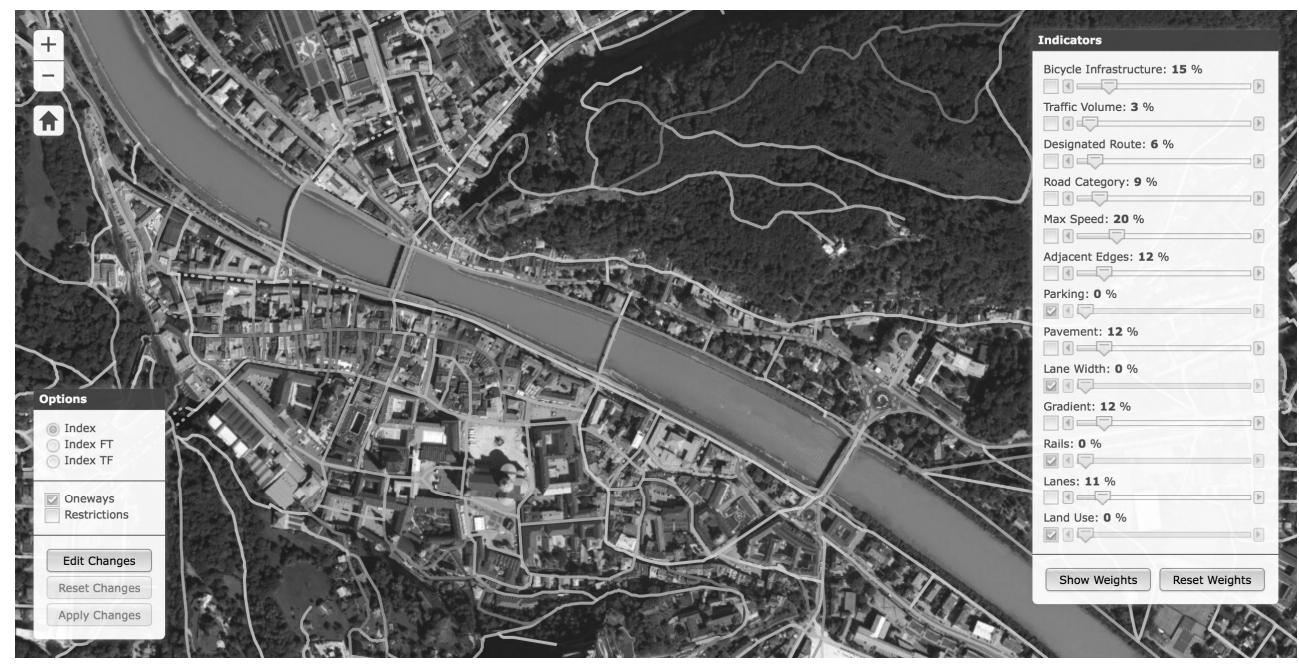

Fig. 4: User interface of the interactive network assessment tool with the "Options" and "Indicators" windows and the colored road network

The options window controls the rendering and the handling of the changes as described before. The indicators window contains the sliders for each indicator and the possibility to export the weights and reset them to the initial values. Because there are numerous indicators, the sliders controlling the weight for a certain indicator can be fixed. The remaining percentage is distributed as maximum value for the other sliders.

\subsection{Results}

The developed tool enables appropriate indicator weightings for different bicycle related purposes to be determined. In the exemplary case of bicycle safety, evaluating the spatial disparities in the road infrastructure, and adapting the weightings to the significant indicators leads to different indicator sets, e.g. for urban and rural environments. These indicator sets allow an overview of the current conditions of the road network. Due to the additionally provided information in the popup window, the index calculation always remains comprehensible and transparent.

Once such an indicator set is found, critical locations in the road network become obvious due to the clear visualization of the index values. Furthermore, the tool is well suited for simulating safety measures and planning bicycle infrastructure. Safety gaps in the network that interrupt well rated bicycle corridors might be found easier by fiddling around with the weightings, maybe by detaching highly weighted indicators first, in order to get a better distribution of the remaining indicators. The provided features may support planners to perceive and countermeasure such gaps by appropriate means. In doing so, corridors could 
be closed at locations where the improvements can be realized at minimum costs. By temporarily simulating selected enhancements on an attributive level, the direct benefit can be displayed, and transparently proven due to a decreasing index value (HARRIS et al. 2013). Fig. 5 shows an exemplary adaption and its impact on the index value and hence the improvement of safety. In combination with a catalogue of measures the necessary steps and incurred costs could be estimated.

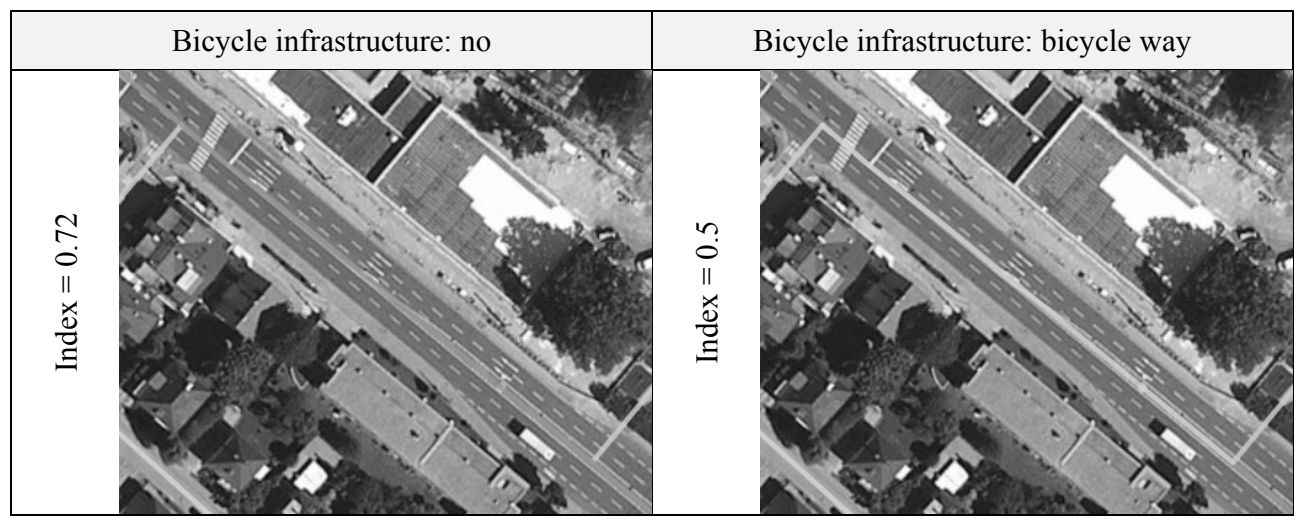

Fig. 5: Adapting the indicator bicycle infrastructure (the color of road segment changes from orange to yellow, showing an improvement of safety)

Because the structure of the used dataset is standardized and homogenous all over Austria, the approach can be applied easily to the whole country. Unfortunately, the comprehensive coverage of the necessary attributes is not guaranteed. The tool itself can be adapted with little effort to fit other use cases as well. Therefore, miscellaneous datasets and/or indicators need to be prepared accordingly and provided online. The sliders need to be assigned to the new indicators and the labels need to be renamed.

\section{Conclusion and Outlook}

The development of the tool has shown that parts of the indicator-based assessment model can be transformed into dynamic processes. The interactive network assessment tool has proven that the possibility of making responsive changes enhances the fields of application, and makes procedures more transparent and comprehensible. The case study has shown that the calculation of the index value with 13 variables can be done fast enough to immediately display the results at least for the area of Salzburg. This affirmation opens doors to further research. In a next step, the value domains inside the indicators could be made dynamic as well, giving full control over all parameters of the original model at any time.

Beyond the implementation of the basic model, the integration of live geoprocessing directly into the tool would bring applications to a new level. Routing applications could be enhanced with optimized profiles or personalized preferences. Routes could be optimized for specified purposes like safety, comfort or scenic beauty, but could also be adapted to any 
other combination of indicators. In the case of cyclists, different profiles could be identified for groups like pupils, racing cyclists, or mountain bikers. A routing application based on variable weightings could immediately change the calculated route, whenever the preferences have been changed. With this extension, experts could optimize specified routing applications by trial and error. In spatial simulations, the mentioned profiles could then be used to simulate agents with diverging behavior (KESTING et al. 2009, BAZZAN \& KLÜGL 2014). The outcomes of such simulations may designate the most frequented edges by cyclists. If the results do not reflect reality, the weightings could be adjusted in an iterative process, in order to find phenomena that have been over- or underrated and to achieve a better description of reality. When the findings of the spatial simulation are adequate, they could again be used to support, for example, a routing application with traffic density values. This would result in alternative route suggestions at highly frequented time frames.

Aside from improving applications, the features provided by an interactive network assessment tool can be used for educational purposes as well. The results of this paper are therefore of interest for a number of parties. Among them are public authorities, traffic planners, smart mobility experts, and also data modelers.

\section{References}

BAZZAN, A. L. C. \& KLÜGL, F. (2014), A review on agent-based technology for traffic and transportation. The Knowledge Engineering Review, 29, 375-403.

Beck, L. F., Dellinger, A. M. \& O'Neil, M. E. (2007), Motor Vehicle Crash Injury Rates by Mode of Travel, United States: Using Exposure-Based Methods to Quantify Differences. American Journal of Epidemiology, 166, 212-218.

ESRI (2014a), Creating web applications with the ArcGIS API for JavaScript. http://resources.arcgis.com/en/help/main/10.2/index.html\#//01540000040n000000 (29.01.2015).

ESRI (2014b), ArcGIS Online - The Mapping Platform for Your Organization. http://www.esri.com/software/arcgis/arcgisonline (29.01.2015).

THE DOJO FoundATION (2015), Dojo - Features. http://dojotoolkit.org/features/ (29.01.2015).

Harkey, D. L., Reinfurt, D. W., Knuiman, M., Stewart, J. R. \& Sorton, A. (1998), Development of the bicycle compatibility index: a level of service concept. Washington D.C., Federal Highway Administration, 116 p.

Harris, M. A., Reynolds, C. C. O., Winters, M., Cripton, P. A., Shen, H., Chipman, M. L., Cusimano, M. D., Babul, S., Brubacher, J. R., Friedman, S. M., Hunte, G., Monro, M., Vernich L. \& TESCHKE K. (2013), Comparing the effects of infrastructure on bicycling injury at intersections and non-intersections using a case-crossover design. Injury Prevention.

Juhra, C., Wieskötter, B., Chu, K., Trost, L., Weiss, U., Messerschmidt, M., MalczyK, A., Heckwolf, M. \& RaschKe, M. (2012), Bicycle accidents - Do we only see the tip of the iceberg?: A prospective multi-centre study in a large German city combining medical and police data. Injury, 43, 2026-2034.

Kesting, A., Treiber, M. \& Helbing, D. (2009), Agents for Traffic Simulation. In: UhrMACHER, A. M. \& WeYns, D. (Eds.), Multi-Agent Systems: Simulation and Applications. CRC Press, Boca Raton, 325-356. 
LOIDL, M. \& ZAGEL, B. (2010), Wie sicher ist sicher? - Innovatives Kostenmodell zur Ermittlung des Gefährdungspotenzials auf Radwegen. In: Strobl, J., BlaschKe, T. \& GrIESEBNER, G. (Eds.), Angewandte Geoinformatik 2010. Beiträge zum 22. AGITSymposium Salzburg. Wichmann, Berlin/Offenbach, 394-403.

LOIDL, M. \& ZAGEL, B. (2014), Assessing bicycle safety in multiple networks with different data models. In: JeKel, T., CAR, A., Strobl, J. \& Griesebner, G. (Eds.), GI_Forum 2014. Geospatial Innovation for Society. Wichmann, Berlin/Offenbach, 144-154.

Lorenc, T., Brunton, G., Oliver, S., Oliver, K. \& OAKley, A. (2008), Attitudes to walking and cycling among children, young people and parents: a systematic review. Journal of Epidemiology and Community Health, 62, 852-857.

Teschke, K., Harris, M. A., Reynolds, C. C. O., Winters, M., Babul, S., Chipman, M., Cusimano, M. D., Brubacher, J. R., Hunte, G., Friedman, S. M., Monro, M., Shen, H., VERnich, L. \& CRIPTON, P. A. (2012), Route Infrastructure and the Risk of Injuries to Bicyclists: A Case-Crossover Study. American Journal of Public Health, 102, 23362343.

Wegman, F., ZhAng, F. \& DiJKstra, A. (2012), How to make more cycling good for road safety? Accident Analysis \& Prevention, 44, 19-29. 